\title{
Effects of Nanoparticles on Non-Darcy Mixed Convective Heat Transfer in Nanofluids over a Shrinking and Stretching Wedge
}

\author{
Hassan Saadi Abdelaal El-dawy ${ }^{1,}$, Rama Subba Reddy Gorla ${ }^{2}$ \\ ${ }^{1}$ High Institute of Engineering and Technology Tod, Luxor, Egypt \\ ${ }^{2}$ Department of Mechanical Engineering, Cleveland State University, Cleveland, USA
}

Email address:

ha_sa3000@yahoo.com (H. S. A. El-dawy)

*Corresponding author

\section{To cite this article:}

Hassan Saadi Abdelaal El-dawy, Rama Subba Reddy Gorla. Effects of Nanoparticles on Non-Darcy Mixed Convective Heat Transfer in Nanofluids over a Shrinking and Stretching Wedge. Applied and Computational Mathematics. Vol. 8, No. 4, 2019 , pp. 70-74. doi: 10.11648/j.acm.20190804.11

Received: June 12, 2019; Accepted: July 3, 2019; Published: September 5, 2019

\begin{abstract}
In this work we studied the effect of nanoparticles on the velocity and heat transfer during the flow of nanofluid in Non-Darcy mixed convection, over a wedge, taking into account of shrinking and stretching of the surface. The governing partial differential equations are converted into ordinary differential equations by means of coordinate transformation. The transformed equations are solved by means of fourth order Runge Kutta method in conjunction with shooting method. The results for the velocity and temperature fields are presented graphically as well as in tabular form. This research is expected to be useful for studying the movement of oil, gas, and water through the oil reservoir or the gas field, in the migration of groundwater and in the purification and purification of water. The friction factor decreases as the nanoparticle concentration increases whereas the heat transfer rate (Nusselt number) increases with nanoparticle concentration. The friction factor and heat transfer rate increase as the suction parameter increases. The friction factor decreases as the wedge angle increases whereas the heat transfer rate (Nusselt number) increases with wedge angle.
\end{abstract}

Keywords: Nanoparticle, Suction, Wedge, Shrinking and Stretching

\section{Introduction}

The conventional fluids in engineering and sciences, such water and oil have primary limitations in enhancing performance of heat transfer due to their thermal conductivities. Nanofluids, these are suspention of nanoparticles in basefluid. Have been proposed recently to improve the thermal conductivity of base fluids. These fluids have distinguished thermal features such as high surface to volume ratio, resulting in their perfect thermal performance. The term nanofluid was introduced by Choi [1]. It is defined as colloidal suspension of nanoparticles dispersed in base fluids. After his study the research in this field has risen exponentially, Parallel theoretical studies are also introduced to modelling of nanofluid behaviours. There are two models are proposed in the literature until now, these are homogeneous models and dispersion models. In this present work we use non homogeneous model for transport phenomena which is proposed by Buongiorno [2], The comprehensive survey of convective transport in the boundary layer flow has been conducted Yocob et al, [3], Mustafa et al, [4], Khan and Aziz [5], Khan and Pop [6], Kuznetsov and Nield, [7].

Mixed convection heat transfer can be observed in many engineering applications such as heat exchangers, cooling of electric equipment's, and heating and cooling of buildings. Many researchers in nanofluid community investigated the merits of dispersing nanoparticles into base fluids to enhance heat transfer in mixed convection settings. The mixed convective flow and heat transfer studied by a number of investigators: Goodari et al. [8], Gumgum et al, [9], Arash Karimipour et al, [10], Kumari and Nath [11], Chamkha and Ahmed [12] and Akbarinia et al, [13].

The efficiencies of thermal devices and systems are related 
to heat transfer rates which in turn depend on the thermal conductivity of the working fluids. Despite considerable previous research and development efforts on heat transfer enhancement, the demand is growing for more efficient and robust heat transfer fluids with significantly higher thermal conductivities than traditional ones. Nanofluids are engineered by suspending nanoparticles with average sizes below $100 \mathrm{~nm}$ in traditional heat transfer fluids such as water, oil, and ethylene glycol. Many interesting properties of nanofluids have been reported in the past decades. Nanofluids have better stability because of the larger surfacearea-to-volume ratio of nanoparticles which overcome differences in density. Therefore, the exploitation of nanofluids opens up the possibilities to improve the efficiency of thermal system while maintaining the existing footprint. Alternatively, nanofluids can provide the same efficiency of the cooling system at smaller and lighter footprint as reduced inventory of heat transfer fluids. Eventually, improved cooling performance and lower manufacturing or operating cost in thermal system are the major advantage derived from the application of nanofluids.

Owing to this stimulating benefit, a large number of researches in the scientific community are being carried out with the goal of realizing various applications of nanofluids. Initial research focused mainly on thermal conductivity, only recently that subsequently researchers delved into other area of interest such as viscosity, specific heat, density, critical heat flux, heat transfer coefficient, entropy and wear resistance. In existing experimental works, nanofluids can be produced by two methods, namely the two-step process and the one-step process. In a typical two-step process, the nanoparticles are first produced in dry powder form and then mixed with the heat transfer fluids. However, these nanofluids are not stable even though the stability could be enhanced by $\mathrm{pH}$ control and surfactant addition. In the onestep process, the synthesis and dispersion of nanoparticles are done at the same time. These nanofluids have better stability due to the weakened Van der Waals force between nanoparticles. Nevertheless, the two-step process is always preferred by the majority of researchers owing to its low setup cost.

The flow of a nanofluid in the vicinity of stagnation point was analyzed by El-Dawy et. al. [14]. Unsteady Flow of a Nanofluid Over a Shrinking/Stretching Porous Wedge was studied by El-dawy and Gorla [15]. Chemical reaction effects on non-Darcy mixed convective heat and mass transfer past a porous wedge were studied by Kandasamy, et, al [16]. Influence of particulate thermophoresis on convection heat and mass transfer was considered by Jize Sui [17]. MHD boundary-layer flow of a micropolar fluid past a flat plate was considered by Nazar, et. el [18]. The mixed convection in a nanofluid past a vertical plate embedded in a saturated porous medium was studied by El-Dawy et. al. [19]. The MHD forced convection flow adjacent to a non-isothermal wedge was considered by Yih [20]. The effect of inclination angle on natural convection in nanofluids was studied by Abu-Nada and Oztop [21].
In this paper, we study the effect of nanoparticles in nanofluids on the surface of a wedge taking into account of shrinking and stretching of the surface.

\section{Mathematical Analysis}

We consider the steady, laminar mixed convective flow of a nanofluid over a wedge. The density variation and viscosity effects are taken into account in the momentum equation and Boussinesq approximation is made. Let the $\mathrm{x}$-axis be taken along the wedge and the $y$ axis normal to the surface of the wedge. We include suction or injection on the surface of the wedge. The governing equations within boundary layer approximation may be written as:

$$
\begin{gathered}
\frac{\partial u}{\partial x}+\frac{\partial v}{\partial y}=0 \\
\rho_{n f}\left(u \frac{\partial u}{\partial x}+v \frac{\partial u}{\partial y}\right)=\mu_{n f} \frac{\partial^{2} u}{\partial y^{2}}+U \frac{d U}{d X}-\mu_{n f}(u-U) \\
F\left(u^{2}-U^{2}\right)+g \beta_{n f}\left(T-T_{\infty}\right) \sin \left(\frac{\Omega}{2}\right)=0 \\
u \frac{\partial T}{\partial x}+v \frac{\partial T}{\partial y}=\frac{\propto_{n f}}{\left(\rho C_{p}\right)_{n f}} \frac{\partial^{2} T}{\partial y^{2}}+\mu_{n f}\left(\frac{\partial u}{\partial y}\right)^{2}=0 \\
u=0, v=-v_{0}, T=T_{w} \text { at } y=0 \\
u=U(x), T=T_{\infty} \text { at } y \rightarrow \infty
\end{gathered}
$$

where $u$ and $\mathrm{v}$ are the velocity components in the $x$ and $y$ directions, respectively, $g$ is the acceleration due to gravity, $\rho_{f}$ is the density of the fluid, $\rho_{s}$ is the density of the nanoparticle; $\beta_{n f}$ is the coefficient thermal expansion; $T, T \mathrm{w}$, and $T \infty$ are the temperature of the fluid inside the thermal boundary layer, the plate temperature and the fluid temperature in the free stream, respectively; $k_{f}$ is the thermal conductivity of the fluid; $k_{s}$ is the thermal conductivity of the nanoparticle, $\mu_{n f}$ is the dynamic viscosity; $C_{p_{f}}$ is the specific heat of the fluid, $C_{p_{s}}$ is the specific heat of the nanoparticle $\Omega$ is the angle of inclination of wedge, and $\mathrm{F}$ is (Forchheimer number).

Proceeding with the analysis, we define the following transformations:

$$
\eta(x, y)=y \sqrt{\frac{(1+m) U}{2 v x}}, \psi(x, y)=\sqrt{\frac{2 U v x}{1+m}} f(x, \eta), U(x)=A x^{m}
$$

Where

$$
u=\frac{\partial \psi}{\partial y} v=-\frac{\partial \psi}{\partial x} \theta=\frac{T-T_{\infty}}{T_{w}-T_{\infty}}
$$

By substituting the transformations defined in (5) into equations (1), (2) and (3) we have:

$$
\begin{gathered}
\mu_{\mathrm{nf}} f^{\prime \prime \prime}+\rho_{\mathrm{nf}} f f^{\prime \prime}+\frac{2}{1+\mathrm{m}}\left(1-f^{2}\right)+\beta_{\mathrm{nf}} \theta \sin \left(\frac{\Omega}{2}\right)-\mu_{\mathrm{nf}}\left(f^{\prime}-1\right)=0 \\
\alpha_{\mathrm{nf}} \theta^{\prime \prime}-\frac{2}{1+m} \theta f^{\prime}+f \theta^{\prime}+\mu_{n \mathrm{f}} f^{\prime \prime 2}=0
\end{gathered}
$$

with transformed boundary conditions: 


$$
\begin{gathered}
f(0)=s, f^{\prime}(0)=h, \theta(0)=1 \eta=0 \\
f^{\prime}(\infty)=1, \theta(\infty)=0 \eta \rightarrow \infty
\end{gathered}
$$

\section{Numerical Solution}

The set of non- linear ordinary Eqs (6) and (7) with boundary conditions (8) have been solved by using the Runge Kutta- Gill method along shooting technique with variables $h<$ 0 is shrinking surface, $h>0$ is sretching surface.

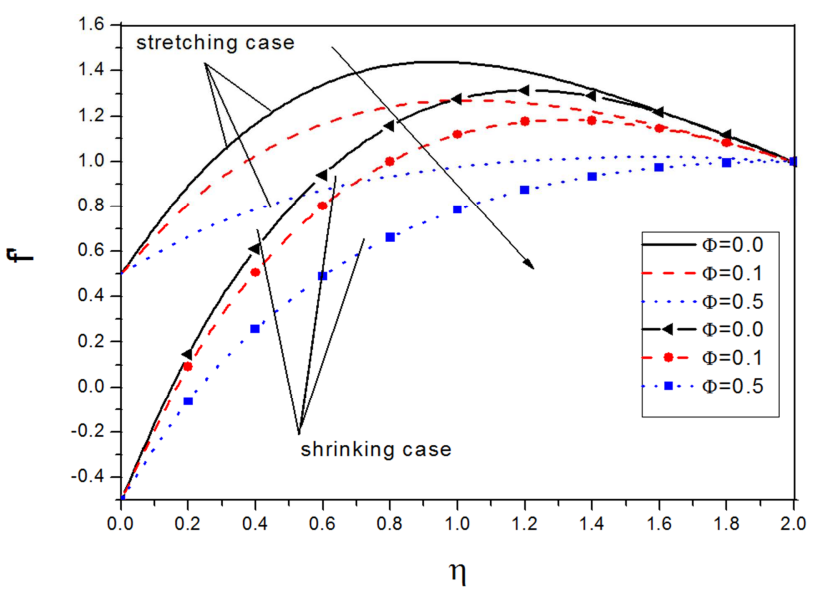

Figure 1. Effect of nanoparticles $\Phi$ in the velocity.

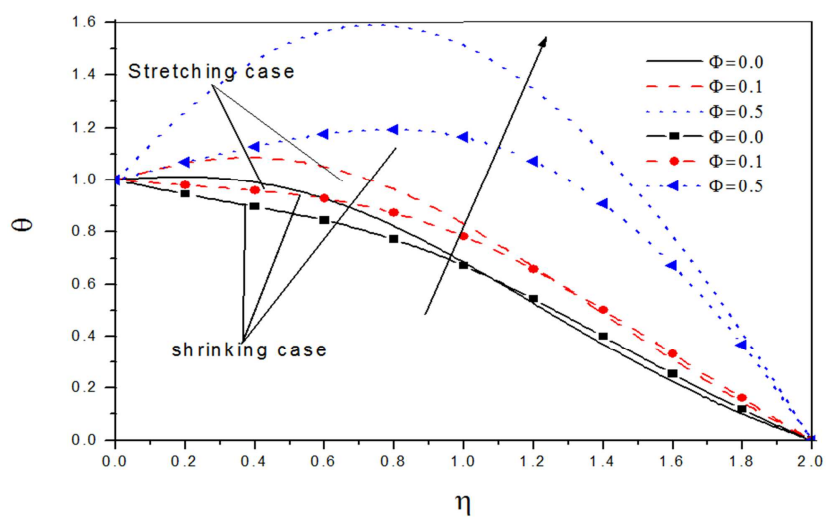

Figure 2. Effect of nanoparticles $\Phi$ in temperature.

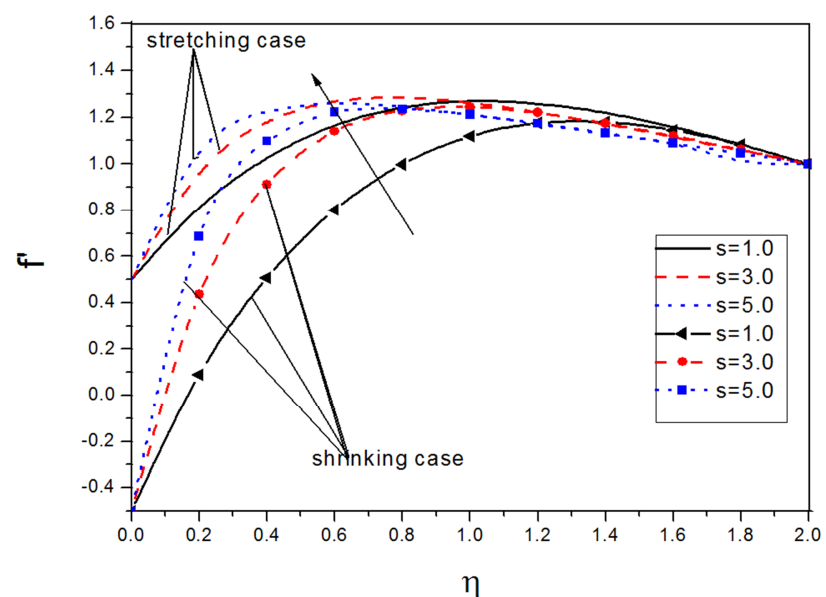

Figure 3. Effect of the nanoparticle suction parameter in the velocity.

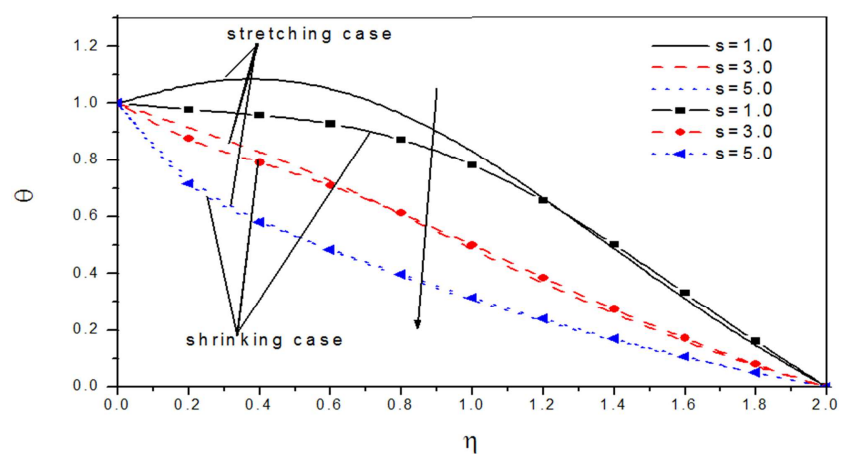

Figure 4. Effect of nanoparticle suction parameter on the temperature profile.

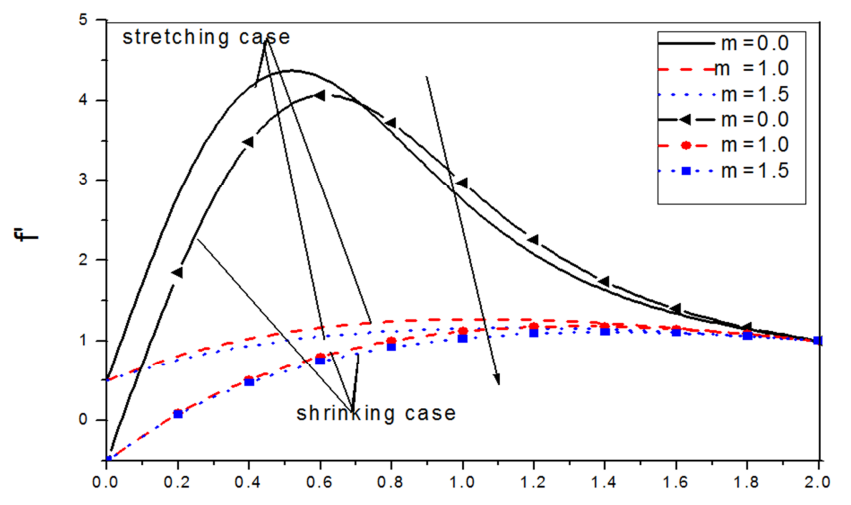

$\eta$

Figure 5. Effects of angle of inclination of wedge on velocity profile.

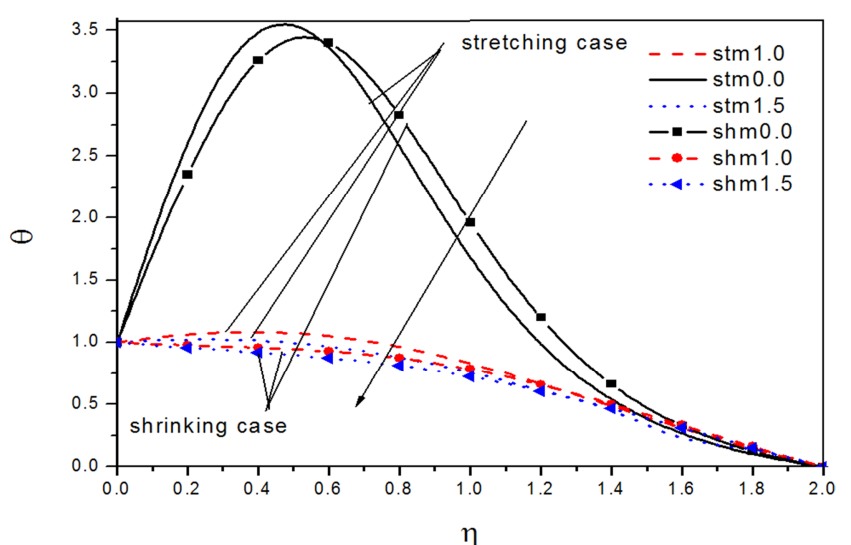

Figure 6. Effects of angle of inclination of wedge on temperature profile.

Table 1. Effect of nanoparticle on skin friction and the Nusselt number.

\begin{tabular}{lll}
\hline $\boldsymbol{\varphi}$ & $\boldsymbol{f}^{\prime \prime}(\mathbf{0})$ & $\boldsymbol{\theta}^{\prime}(\mathbf{0})$ \\
\hline 0.0 & 2.2854 & 0.1051 \\
0.1 & 1.8267 & 0.4006 \\
0.5 & 0.9666 & 1.4462 \\
\hline
\end{tabular}

Table 2. Effect of suction parameter on skin friction and the Nusselt number.

\begin{tabular}{lll}
\hline $\boldsymbol{s}$ & $\boldsymbol{f}^{\prime \prime}(\mathbf{0})$ & $\boldsymbol{\theta}^{\prime}(\mathbf{0})$ \\
\hline 3.0 & 3.1514 & -0.4793 \\
5.0 & 4.4224 & -1.9087 \\
7.0 & 5.5979 & -3.5382 \\
\hline
\end{tabular}


Table 3. Effect of measure angle on skin friction and the Nusselt number

\begin{tabular}{lll}
\hline $\boldsymbol{m}$ & $\boldsymbol{f}^{\prime \prime}(\mathbf{0})$ & $\boldsymbol{\theta}^{\prime}(\mathbf{0})$ \\
\hline 1.0 & 2.2854 & 0.0003 \\
2.0 & 2.2549 & -0.3901 \\
4.0 & 1.4644 & -9173 \\
\hline
\end{tabular}

\section{Result and Discussion}

The effect of nanoparticle on nanofluids, which, passed over a shrinking and stretching wedge.

We see the results appear from the figures:

Figure 1 shows the effect of nanoparticle concentration on the velocity distribution within the boundary layer. We see from Figure 1 that as the nanoparticle concentration increases, in each case stretching and shrinking, the velocity decreases. Figure 2 shows the effect of nanoparticle concentration on the temperature distribution within the boundary layer. We see from Figure 2 that as the nanoparticle concentration increases, in each case stretching and shrinking, the temperature increases.

Figure 3 shows that as the suction parameter increases in each case, stretching and shrinking, the velocity increases. Figure 4 shows that as the suction parameter increases in each case, stretching and shrinking, the temperature decreases.

Figure 5 shows the effect of wedge angle on velocity distribution. We notice that as the wedge angle increases, in each case stretching and shrinking, the velocity decreases. Figure 6 shows the effect of wedge angle on temperature distribution. We notice that as the wedge angle increases, in each case stretching and shrinking, the temperature decreases.

Table 1 shows that the friction factor decreases as the nanoparticle concentration increases whereas the heat transfer rate (Nusselt number) increases with nanoparticle concentration.

Table 2 shows that the friction factor and heat transfer rate increase as the suction parameter increases.

Table 3 shows that the friction factor decreases as the wedge angle increases whereas the heat transfer rate (Nusselt number) increases with wedge angle.

\section{Conclusion}

In this work we presented a boundary layer analysis to study the effects of nanoparticles on the behavior of fluid flow over shrinking and stretching wedge in non-Darcy medium. This research is expected to be useful for studying the movement of oil, gas, and water through the oil reservoir or the gas field, in the migration of groundwater and in the purification and purification of water. The friction factor decreases as the nanoparticle concentration increases whereas the heat transfer rate (Nusselt number) increases with nanoparticle concentration. The friction factor and heat transfer rate increase as the suction parameter increases. The friction factor decreases as the wedge angle increases whereas the heat transfer rate (Nusselt number) increases with wedge angle.

\section{References}

[1] S. U. S. Choi, Enhancing thermal conductivity of fluids with nanoparticles in developments and Applications of NonNewtonian Flows. D. A Springer and H. P. wang, Eds., ASME. 66 (1995), 99-105.

[2] J. Buongiorno, Convective Transport in Nanofluid, Journal of Heat Transfer, 128 (2006), 240-250.

[3] Nor Azizah Yacob, Anuar Ishak, Roslinda Nazar, Ioan Pop, Falkner-Skan problem for a static and moving wedge with prescribed surface heat flux in a nanofluid, International Communications in Heat and Mass Transfer. 38 (2011), 149153.

[4] M. Mustafa, T. Hayat, I. Pop, S. Asghar, S. Obaidat, Stagnation-point flow of a nanofluid towards a stretching sheet, International Journal of Heat and Mass Transfer, 54 (2011), 5588-5594.

[5] W. A. Khan, I. Pop, Boundary-layer flow of a nanofluid over a stretching sheet, International Journal of Heat and Mass Transfer, 53 (2010), 2477-2483.

[6] W. A. Khan, A. Aziz, Natural convection flow of a nanofluid over a vertical plate with a uniform surface heat flux, International Journal of Thermal Sciences, 7 (2011), 12071214.

[7] A. V. Kuznetsov, D. A. Nield, Natural convective boundarylayer flow of a nanofluid past a vertical plate, International Journal of Thermal Sciences, 49 (2010), 243-247.

[8] M. Goodarzi, M. R. Safaei, K. Vafai, G. Ahmadi, M. Dahari, S N. Kazi, N. Jomhari, Investigation of nanofluid mixed convection in a shallow cavity using a two phase mixture model, International Journal of Thermal Sciences, 75 (2014), 204-220.

[9] S. Gumgum, M. Tezer-Sezgin, DRBEM solution of mixed convection flow of a nanofluids in enclosures with moving walls, Journal of Computational and Applied Mathematics, 259 (2014), 730-740.

[10] Arash Karimipour, Mohammad Hemmat Esfe, Mohammad Reza Safaei, Davood Toghraie Semiromi, Saeed Jafari, S. N. Kazi, Mixed convection of Copper-Water nanofluid in a Shallow inclined lid driven cavity using the Lattice Boltzmann Method, Physica A, 402 (2014), 150-168.

[11] M. Kumari, G. Nath, Unsteady incompressible boundary layer flow over a rotating sphere, Journal of Applied Mechanics, 49 (1982), 234-236.

[12] Ali J. Chamkha, sameh E. Ahmmed, Unsteady MHD Heat and Mass Transfer by Mixed Convection Flow in the Forward Stagnation Region of a Rotating Sphere at Different Wall Conditions, Chemical Engineering Communication, 199 (2012), 122-141.

[13] A. Akbarinia, A. Behzadmehr, Numerical study of laminar mixed convection of a nanofluid in a horizontal curved tubes, Applied Thermal Engineering, 27 (2007), 1327-1337.

[14] H. A. El-dawy. et. al Stagnation Point Thermal Boundary Layer Flow Towards a Stretching/Shrinking Sheet in a Nanofluid Journal of Nanofluids Vol. 2, pp. 1-5, 2013. 
[15] H. A. El-dawy, r. gorla Unsteady Flow of a Nanofluid Over a Shrinking/Stretching Porous Wedge Sheet in the Presence of Solar Radiation Journal of Nanofluids Vol. 7, pp. 1-9, 2018Journal of Nanofluids.

[16] R. Kandasamy reaction effects on non-Darcy mixed convective heat and mass transfer past a porous wedge with variable viscosity in the presence of suction or injection Nuclear Engineering and Design 238 (2008) 2699-2705.

[17] Jize Sui Influence of particulate thermophoresis on convection heat and mass transfer in a slip flow of a viscoelasticity-based micropolar fluid International Journal of Heat and Mass Transfer 119 (2018) 40-51.

[18] Roslinda Nazar, et. al MHD boundary-layer flow of a micropolar fluid past a wedge with constant wall heat
Communications in Nonlinear Science and Numerical Simulation 14 (2009) 109-118.

[19] H. A El-Dawy, et, al. Mixed Convection in a Nanofluid Past a Vertical Plate in a Saturated Porous Medium Journal of Nanofluids Vol. 3, pp. 1-4, 2014.

[20] Yih KA. MHD forced convection flow adjacent to a nonisothermal wedge. Int Common Heat Mass Transfer 1999; 26: 819-27.

[21] E. Abu-Nada, H. F. Oztop, Effect of inclination angle on natural convection in enclosures filled with $\mathrm{Cu}$-water nanofluid, Int. J. Heat Fluid Flow 30 (2009) 669-678. 